\title{
An Analysis of Physicochemical Properties for Drugs of Natural Origin
}

David Camp,$^{*}{ }^{\dagger}$ Agatha Garavelas, ${ }^{\ddagger}$ and Marc Campitelli ${ }^{*, \mathcal{S}}$

† School of Environment, Griffith University, Nathan Campus QLD 4111 Australia

${ }^{\ddagger}$ Eskitis Institute, Griffith University, Nathan Campus QLD 4111 Australia

${ }^{\S}$ QIMR Berghofer Medical Research Institute, Herston QLD 4006 Australia 
ABSTRACT: The impact of time, therapy area, and route of administration on 13 physicochemical properties calculated for 664 drugs developed from a natural prototype was investigated. The mean values for the majority of properties sampled over five periods from pre-1900 - 2013 were found to change in a statistically significant manner. In contrast, lipophilicity and aromatic ring count remained relatively constant suggesting that these parameters are the most important for successful prosecution of a natural product drug discovery program if the route of administration is not focused exclusively on oral availability. An examination by therapy area revealed that anti-infective agents had the most differences in physicochemical property profiles compared with other areas, particularly with respect to lipophilicity. However, when this group was removed, the variation between the mean values for lipophilicity and aromatic ring count across the remaining therapy areas was again found not to change in a meaningful manner further highlighting the importance of these two parameters. The vast majority of drugs with a natural progenitor were formulated for either oral and/or injectable administration. Injectables were, on average, larger and more polar than drugs developed for oral, topical and inhalation routes. 
An examination of drug-likeness in the context of oral bioavailability was first reported by Lipinski, Lombardo, Dominy, and Feeney in a pivotal analysis that identified four physicochemical properties common to the $90^{\text {th }}$ percentile of approximately 2500 drugs and candidate drugs reaching phase II clinical trials. ${ }^{1}$ Their findings were distilled into the now well-known "rule of five” (Ro5): molecular weight (MW) < 500 Da; H-bond donors (HBD) < 5; H-bond acceptors (HBA) < 10 and; the calculated logarithm of the 1-octanol/water partition coefficient $(\mathrm{cLogP})<5 .^{1}$ At its core, the Ro5 is a set of guidelines that identifies key properties assumed to be good indicators of oral absorption while, at the same time, highlighting potential bioavailability issues if two or more violations occur. In this sense, the Ro5 does not define drug-like chemical space per se but is rather a predictor for oral bioavailability. ${ }^{2}$

Importantly, the Ro5 also contains a significant caveat, i.e. “compound classes that are substrates for biological transporters are exceptions to the rule."1 The authors observed that certain classes like antibiotics, antifungals, vitamins, and cardiac glycosides tended to violate the Ro5. While there is a clear link between these classes and natural compounds, it must be noted that natural products were not explicitly captured by this qualification. There has been considerable debate whether drugs permeate a cell exclusively via a carrier-mediated mechanism ${ }^{3,4}$ or if this co-exists with passive diffusion. ${ }^{5,6}$ In the absence of any definitive evidence for an active transport mechanism, it has been suggested that structurally complex natural products like paclitaxel (1) and sirolimus (2) penetrate cells via passive diffusion despite their high MW and other violations of the Ro5. ${ }^{7}$ This may be achieved by a judicious positioning of $\mathrm{H}$-bond donors and acceptors to form intramolecular $\mathrm{H}$-bonds that limit molecular flexibility and reduces the polar surface area (PSA). ${ }^{7,8}$ 

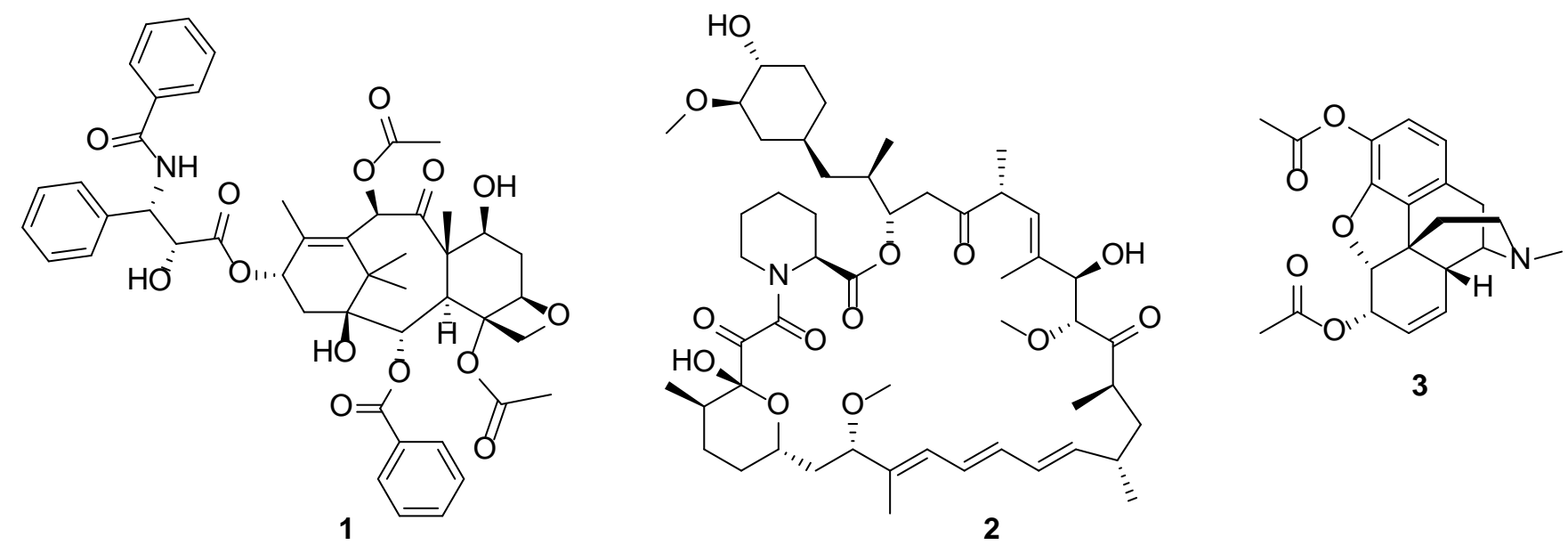

Since Lipinski and co-workers' seminal paper, medicinal chemists have become increasingly aware of causal relationships between physicochemical properties of potential drug candidates and absorption, distribution, metabolism, excretion, and toxicity (ADMET) prediction models. ${ }^{9}$ Subsequent studies have identified additional properties considered to be useful predictors of oral bioavailability, e.g., Veber and co-workers ${ }^{10}$ used a data set of 1100 compounds and found that those with fewer than 10 rotatable bonds $(\mathrm{RTB})$ and PSA $<100 \AA^{2}$ were more likely to achieve greater than $20 \%$ oral bioavailability in rats. Lu and co-workers ${ }^{11}$ later cautioned against generalizing cut-off points after they showed that values depended on the calculation method and therapeutic area. One approach to circumvent rigid cut-offs was proposed by the multiparameter quantitative estimate of drug-likeness which employs seven commonly used parameters - the four Ro5 properties plus RTB, PSA, aromatic ring count (AROM), and a structure alert - to facilitate ranking of compounds for development. ${ }^{12}$

The AROM descriptor has received greater attention after Ritchie and Macdonald published an analysis of GlaxoSmithKline compounds that outlined how an increase of AROM negatively impacted on properties like aqueous solubility, serum albumin binding, and hERG inhibition. ${ }^{13}$ A number of aromatic descriptors were subsequently introduced into the literature and have been the subject of a recent review. ${ }^{14}$ Around the same time, Lovering et al. reported that two simple shape-based descriptors, tetrahedral carbon atom fraction (Fsp3) and the number of stereogenic carbon atoms (Chiral), correlated with aqueous solubility and the successful passage of compounds from discovery, 
through clinical testing, to drugs. ${ }^{15}$ Indeed, the authors proposed that the shift to combinatorial chemistry libraries comprised of predominately achiral, aromatic compounds may have been a causal factor for the high failure rate observed at the onset of the high-throughput era. ${ }^{15}$ Not surprisingly, a decrease in AROM and concomitant increase in Fsp3/Chiral correlated with an increase in aqueous solubility, ${ }^{15}$ described by $\operatorname{LogS}_{7.4}{ }^{16}$ in this study.

LogD [the calculated logarithm of the 1-octanol/water partition coefficient of both the ionized and neutral (unionized) forms of a compound at a fixed $\mathrm{pH}$, typically the physiological $\mathrm{pH}=7.4]^{17}$ is another important parameter given that the degree of ionization of a compound can dramatically effect crucial physicochemical properties such as solubility, lipophilicity, and permeability. ${ }^{18}$ According to $\mathrm{pH}$-partition theory, it is the neutral species that preferentially permeates through the gastrointestinal and other lipid membranes via passive diffusion. Additionally, the theory correlates lipophilicity to both the rate and degree to which a compound is absorbed and permeates a membrane. ${ }^{18}$

Along with the above descriptors, two other readily calculated properties, ring count (RNG) and percent polar surface area (\%PSA), ${ }^{19}$ were also used in this investigation to help gauge molecular complexity and the balance between polar and nonpolar properties, respectively. Combined, these 13 properties provide a quantitative measure of drug-likeness.

It is also worth noting that a somewhat more lateral approach was proposed by Leeson and Davis to identify physicochemical properties that could guide library design and subsequent drug development. ${ }^{19}$ Their hypothesis that the physicochemical parameters that remained consistent over the relatively short history of modern drug discovery were more likely to also be the most important, was simple yet profound. An analysis of 864 oral drugs approved prior to 1983 compared with 329 between 1983 2002 revealed that the median cLogP, \%PSA and HBD were virtually unaffected while other physicochemical properties like MW and HBA increased steadily in a statistically significant manner. ${ }^{19}$ 
A follow-up investigation was published a few years later by Leeson, St-Gallay, and Wenlock who tested the "time-stable" hypothesis ${ }^{20}$ after it was reported that the ionization state of a compound had a marked effect on expected ADMET properties ${ }^{21}$ and the number of aromatic rings could affect development of a lead compound en route to the drug. ${ }^{13,15}$ To address these concerns, the authors examined the impact of ion class (for acidic, basic, and neutral drugs), and aromatic content, on physicochemical properties over 12 intervals. ${ }^{20}$ They found that, with the exception of acidic drugs approved after 1990, the mean values for cLogP, \%PSA, and HBD had remained relatively constant since the 1960s. The number of HBA in drugs, on the other hand, increased over time but no timedependent trends were apparent for various shape-based parameters (Fsp3, Chiral, and two descriptors for aromaticity). Ultimately, the authors concluded that, in the case of oral drugs at least, the control of lipophilicity, HBD, 3-dimensionality, and aromaticity are important success factors. ${ }^{20}$

While considerable effort has been dedicated towards understanding the physicochemical requirements of leads and drugs based on predominately synthetic motifs in the high-throughput paradigm, ${ }^{1,2,22-24}$ far less attention has been directed towards their naturally occurring counterparts in the same setting. ${ }^{25,26}$ Ganesan first analyzed a specific set of 24 natural product progenitors discovered since 1970 that led to new drug approvals over the 25-year interval from $1981-2006 .^{8}$ Sometime later, Koehn identified five additional structures between 2007 - 2012 using the same criteria. ${ }^{27}$ These compounds had not been used as leads in any previous drug development program and, as such, represented the total number of unique natural product starting points for drugs that were marketed from 1981 - 2012.

The lack of a more complete analysis for drugs developed from naturally occurring prototypes is unfortunate as, according to a series of reviews by Newman and co-workers, ${ }^{28-31} \sim 39 \%$ of all smallmolecule new molecular entities (NMEs) in the 30-year period to December 2010 were either an unmodified natural compound, an analogue of the natural substance, or a synthetic molecule inspired by a natural product. Clearly, natural compounds are major contributors to drug discovery. ${ }^{32,33}$ However, 
while the development of synthetic drugs has been well documented, the prosecution of a natural product that is not lead- or drug-like according to conventional wisdom could be problematic to progress in a work stream that has evolved around libraries based on non-natural motifs, and biased towards oral bioavailability.

Hence, in an effort to better understand what features, if any, may be important in drugs developed from a natural prototype, we compiled a comprehensive data set of both leads and drugs, and calculated the physicochemical properties for both groups. We envisaged that an analysis of the data may highlight essential properties that could ultimately be used to better inform the prosecution of natural products in drug discovery.

\section{RESULTS AND DISCUSSION}

Identification of Drugs Developed from a Natural Prototype. Newman and co-workers' series of reviews provides detailed information as to the origin of marketed drugs launched from $1981-2010 .^{28-}$

31 Butler has also delivered rolling updates over the past 14 years covering the contributions made specifically by natural product leads towards new drug approvals and candidates in clinical trials. ${ }^{34-36}$ Although their combined coverage of therapeutics based on natural pharmacophores was very comprehensive from 1981 onwards, we felt inclusion of drugs developed from a natural progenitor prior to this was important as a limited (e.g. 20-year) window may not necessarily capture significant trends in physicochemical properties. For example, an analysis by Vieth and co-workers of therapeutics launched between 1982 - 2002 suggested that minimal changes were observed in the median MW and cLogP. ${ }^{37}$ However, a broader examination by Leeson and Davis (vide infra) that grouped drugs chronologically and then analyzed property data over a longer time frame, led to a different conclusion. $^{19}$

Throughout this investigation, we have used five intervals to analyze and compare the property changes of drugs derived from natural progenitors over time: the beginnings of the pharmaceutical industry in 
which purified compounds were used to treat various ailments from pre-1900 - 1940 (labeled 1900 1940 in the property plots); $3 \times 20$-year bins from $1941-2000$ which equate roughly to the dawn of the modern era of drug discovery and concomitant rise of organic chemistry particularly with respect to the total synthesis of natural products (1941 - 1960), the adolescence of organic chemistry synthesis (1961 - 1980), and the maturation of organic synthesis and emergence of combinatorial chemistry (1981 2000); $;^{38}$ and finally, the post millennium period from $2001-2013$.

Before proceeding further, it is politic to remember that the history of ethical drug development has changed considerably from one in which a new product could be supplied direct to the public with minimal (or no) preclinical/clinical testing to one that is now underpinned by stringent evaluation in many countries. Thus, despite the fact some drugs have entered the public sphere without the same level of scrutiny as others; we nevertheless opted to include earlier products. To this end, therapeutics from the fledgling pharmaceutical industry were not omitted provided evidence of their use was obtained or, more germanely, they were still employed legally in some jurisdiction today. By way of example, diamorphine (heroin, 3), a drug first synthesized in the 1870s and then marketed in the late $1890 \mathrm{~s}^{39}$ before its addictive properties prompted some governments to prohibit its use, is still used for the relief of severe pain in the UK. ${ }^{40}$ Finally, four filters were applied so that the final data set of 664 drugs was reflective of small-molecules developed from biodiscovery (Table S1, Supporting Information A). The four filters excluded: 1) drugs based on endogenous ligands in humans; 2) therapeutics with MW > 2000 Da; 3) dietary supplements (e.g. vitamins and essential fatty acids); and 4) molecules that co-ordinate or covalently bind to a metal.

During preparation of this manuscript, Patridge and co-workers reported a complementary data set of all FDA-approved NMEs derived from natural products. ${ }^{41}$ The main differences between the two sets are that while ours captured additional drugs from countries outside the USA, it did not incorporate others on account of the above filters, e.g., nearly one-half of the 547 compounds in the Patridge set were 
obtained from mammals from which steroids were a major contributor. Dietary supplements like folic acid and high MW therapeutics such as heparin were also included in contrast to our study.

Classification System for Drugs of Natural Origin. To facilitate downstream analysis, drugs developed from a natural prototype were classified into one of three categories already articulated by Wilson and Danishefsky: ${ }^{42,43}$

- The natural product lead progresses to the drug unchanged $(\mathrm{N})$;

- The drug is based on a naturally occurring compound (NB);

- The drug is inspired by a natural product (NI).

The $\mathrm{N}$ descriptor represents the most direct route from lead to pharmaceutical. In effect, the lead possesses all of the potency, selectivity, and pharmacokinetic properties of a clinically useful therapeutic such that no further modification is required. ${ }^{42}$ It is immaterial whether the marketed natural product is obtained by isolation from an organism, total synthesis, or semisynthesis from a naturally occurring precursor.

The NB classification reflects the fact that unmodified natural products do not automatically qualify as the best drugs and are typically optimized, usually via organic synthesis reactions, to yield a therapeutic with improved pharmacological properties. ${ }^{44}$ Analogues generated using this approach are considered to be natural product-based. ${ }^{43}$ For the purposes of this investigation, all drugs in the NB category contain a substructure with the same atomic ensemble and sequence present in the natural motif.

Drugs in the NI category contain synthetic motifs that have been inspired in some way by the innate wisdom of Nature. In broad terms, NI-type compounds would cover cases where a pharmacophore, scaffold, or skeleton from the drug prototype was modified into a non-natural (i.e. synthetic) motif. A particularly good illustration is the penem class of compounds. While no penems have yet been found in Nature, it is patently obvious that faropenem (4) was inspired by the closely related carbapenem and 
penam core structures depicted in thienamycin (5) and penicillin G (6), respectively (Figure 1). The lead:drug pair of halichondrin B (7) and eribulin (8) is an example where the natural pharmacophore was excised from the natural product, and the skeleton then subtly altered from a tetrahydropyran ester to an oxomethyl tetrahydrofuran. Similarly, mevastatin (9):rosuvastatin (10)/atorvastatin (11) show how the pharmacophore can be dissected and relocated to a support structure that is completely unlike that of the natural progenitor. Modification of the natural framework of epothilone B (12), albeit by a single atom to afford ixabepilone (13), demonstrates how a therapeutic can also be "inspired” according to the above definition. 


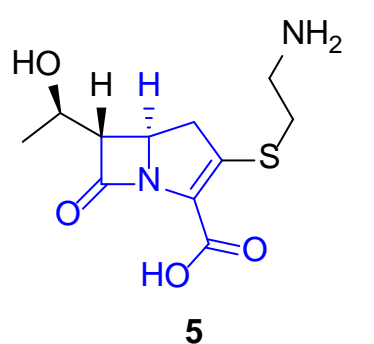

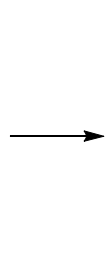

$\mathrm{HO}$<smiles>CC(O)[C@H]1C(=O)N2C(C(=O)O)=C(C3CCCO3)S[C@H]12</smiles>

4

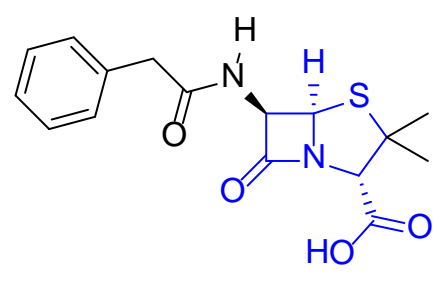

6

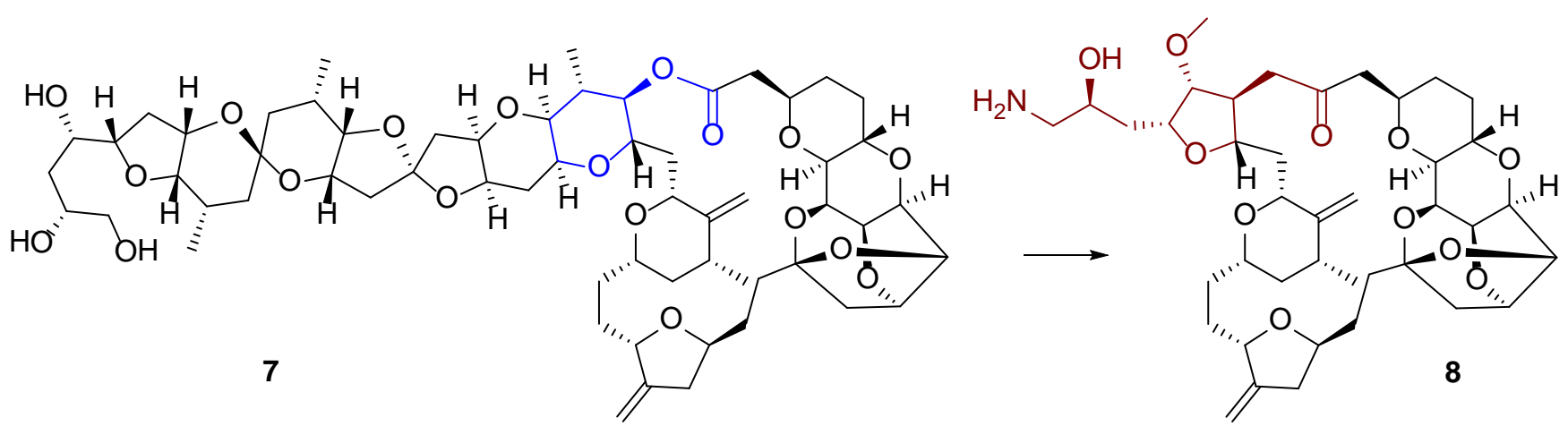<smiles>CC(C)c1nc(N(C)S(C)(=O)=O)nc(-c2ccc(F)cc2)c1/C=C/[C@H](O)C[C@H](O)CC(=O)O</smiles>

10<smiles>CC[C@H](C)C(=O)O[C@H]1CCC=C2C=C[C@H](C)[C@H](CCC3C[C@H](O)CC(=O)O3)[C@H]21</smiles>

9

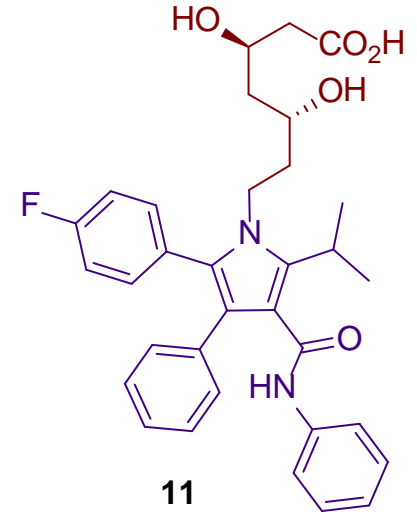

11

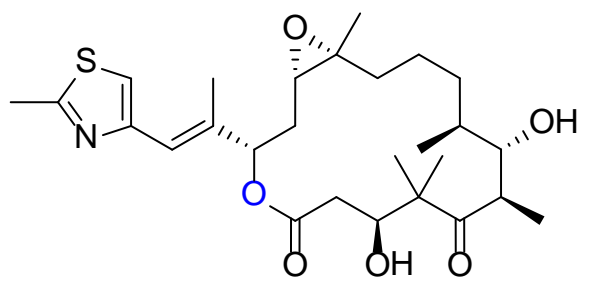

12

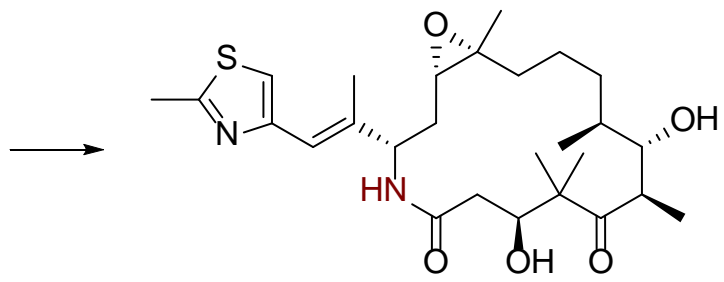

13

Figure 1. Examples of natural product leads and their corresponding drugs in the NI category. Natural substructures or atoms that changed in the progenitor are shown in blue. Modifications that carried through to the therapeutic are depicted in dark red. The purple substructures are synthetic supports to which a pharmacophore was relocated. 
Finally, it was not always possible to discern a unique progenitor for drugs based on, or inspired by, a natural motif if there was more than one candidate. In many situations the simplest base structure was taken as the lead. Thus, tetracycline was selected as the prototype for the tetracycline class of antibiotics. On other occasions a lead molecule was chosen because of some underlying rationale as was the case for penicillin G (6) where chemical or enzymatic processes afforded the 6aminopenicillanic acid core structure from which the vast majority of penicillins were ultimately derived.

Influence of Time on Physicochemical Properties. The origin of the 664 pharmaceuticals in this study could be traced to 196 unique natural prototypes that span a period of almost two centuries, beginning with morphine (1804) and currently sitting at epothilone B (12) (1995), if the year they were first isolated is used as a guide to chronological discovery (Table S1, Supporting Information A). No additional progenitors discovered from 1997 onwards had translated into a therapeutic by the end of 2013. This could be a reflection of the 8 - 15 year timeline from discovery to approval, although Butler has noted a "worrying” development in which only three drug candidates that, in some way utilized a novel natural pharmacophore identified since 2001, actually progressed to clinical trials. ${ }^{36}$ Our data also highlight this concern as shown in Figure S1, Supporting Information B where the absolute contribution from N, NB, and NI drug categories has decreased markedly since the 1961 - 1980 bin to the most recent by approximately $70-80 \%$.

Figures 2 (for Ro5 parameters) and S2, Supporting Information B indicate that an overall upward trend in the mean and median values for MW, HBD, HBA, Fsp3, Chiral, RTB, and PSA was apparent for the set of 664 drugs if the period they were patented/launched was used as a chronological gauge. Despite a rise in these properties, only the mean MWs for the two most recent bins (498.79 for 1981 - 2000 and 639.68 for 2001 - 2013) were at the cusp/violated a Ro5 parameter. A sustained downward movement was evident for $\operatorname{LogS}_{7.4}$; however the lowest mean value of -3.3 is still above -4.0 , generally considered 
the threshold for an orally available drug. In contrast, the mean and median values for cLogP, $\log \mathrm{D}_{7.4}$, \%PSA, RNG, and AROM appear from the property plots to have remained relatively constant.
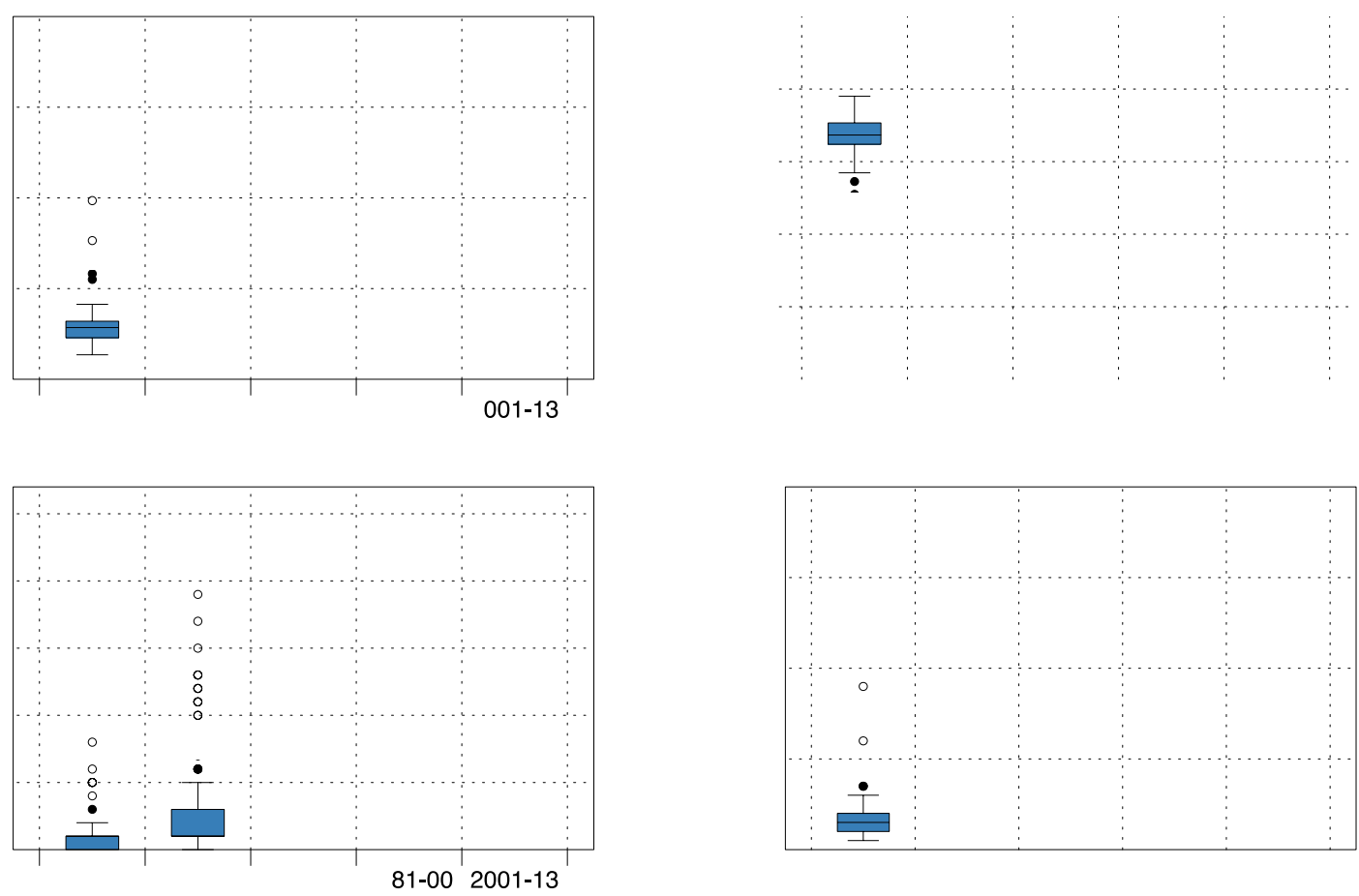

Figure 2. Ro5 property profiles with respect to time for the set of 664 drugs developed from a natural prototype. The interval labeled “1900-40” also includes drugs used prior to 1900. The Tukey plots can be interpreted as follows: the horizontal black line within the box represents the median value, the box represents the interquartile range (IQR) encompassing the middle $50 \%$ of data around the median (i.e. Q3 - Q1). The whiskers represent 1.5 × IQR and the closed and open points represent outliers $(>1.5 \times$ IQR) and extreme outliers (> $3 \times \mathrm{IQR})$, respectively. The yellow circles represent the mean value.

Thus, in an effort to properly discern those parameters that varied the least between the different intervals, a one-way analysis of variance (ANOVA) at a 95\% level of confidence was used to test the null hypothesis that all group means were equal. A $p$-value $>0.1$ indicates there is no evidence to support the alternative hypothesis (i.e. at least two population means are significantly different) and, for that property, there is minimal variation between the group means. At this level of confidence, only cLogP and AROM returned a $p$-value $>0.1$, suggesting that these two properties are important markers 
for the successful development of drugs from a natural prototype. The presence of lipophilicity is not unexpected given it has already been identified in a study of oral drugs, ${ }^{19}$ and its role in influencing drug potency, ${ }^{9}$ pharmacokinetics, ${ }^{45}$ promiscuity, ${ }^{46}$ and toxicity ${ }^{47,48}$ is well established. ${ }^{49}$

The identification of AROM is, likewise, unsurprising given a recent review on the effect of aromatic ring count indicates that increasing the number of benzenoid rings in particular can have a detrimental effect on human bioavailability. ${ }^{50}$ Aqueous solubility was shown to decrease with increasing AROM even when cLogP was kept relatively constant suggesting aromatic ring count is influencing solubility independently of lipophilicity. ${ }^{13}$ Since higher numbers of carboaromatic rings increase lipophilicity (and often potency), but at the expense of solubility, it has been suggested that their numbers in a drug be limited to three. ${ }^{13}$

Similar results to identify properties of fundamental importance were obtained using the two sample ttest employed by Leeson and Davis (Table S1, Supporting Information B) after the set of drugs was divided into two groups around roughly the same transition point (1981 compared with 1983). The differences in mean and median values for 13 physicochemical properties were calculated, and those where both scores were within $\pm 5 \%$, and the $p$-value $>0.1$, was cLogP. The small percentage difference in mean and median scores for cLogP between the two groups in this analysis is quite remarkable given the time scale is over 100 years. The combined results from the t-test and ANOVA suggest that lipophilicity is the single most important property to consider in the context of natural product drug discovery. Notably, Leeson and Davis also found that the mean and median values for HBD and \%PSA of oral drugs changed by less than 5\%, prompting them to also consider these properties of fundamental importance alongside lipophilicity. ${ }^{19}$ The present study suggests that HBD and \%PSA may be less critical in the context of natural products if routes other than oral administration are considered. 
Influence of Drug Category on Physicochemical Properties. Consistent with the analysis of Patridge et al., ${ }^{41}$ we have also observed an decrease in relative terms of unmodified N-type drugs from 40 to less than 20\% (Figure S1, Supporting Information B). This was accompanied by a concomitant rise in therapeutics from the NB-category which has remained above $60 \%$ since the 1960 s. Given the relative contribution of drugs inspired by a natural compound has barely changed in over 100+ years, it is interesting to speculate whether NI-type compounds will ever significantly impact in both absolute and relative terms, or if changes to the natural motif will remain limited so that their present (low and flat) trajectory is maintained. Recent examples such as eribulin (8), rosuvastatin (10), and ixabepilone (13) suggest that there is certainly scope for an increased contribution.

Individual property profiles were found to be highly dependent on the drug category. Generally, the IQR was broadest for N-type compounds, narrowest for NI drugs, with NB pharmaceuticals lying somewhere in between (see Figure 3 for Ro5 parameters and Figure S2, Supporting Information B for the full set of 13 properties). As can also be seen in Figure 3, NI drugs were also positioned more comfortably inside Lipinski space than the other categories.
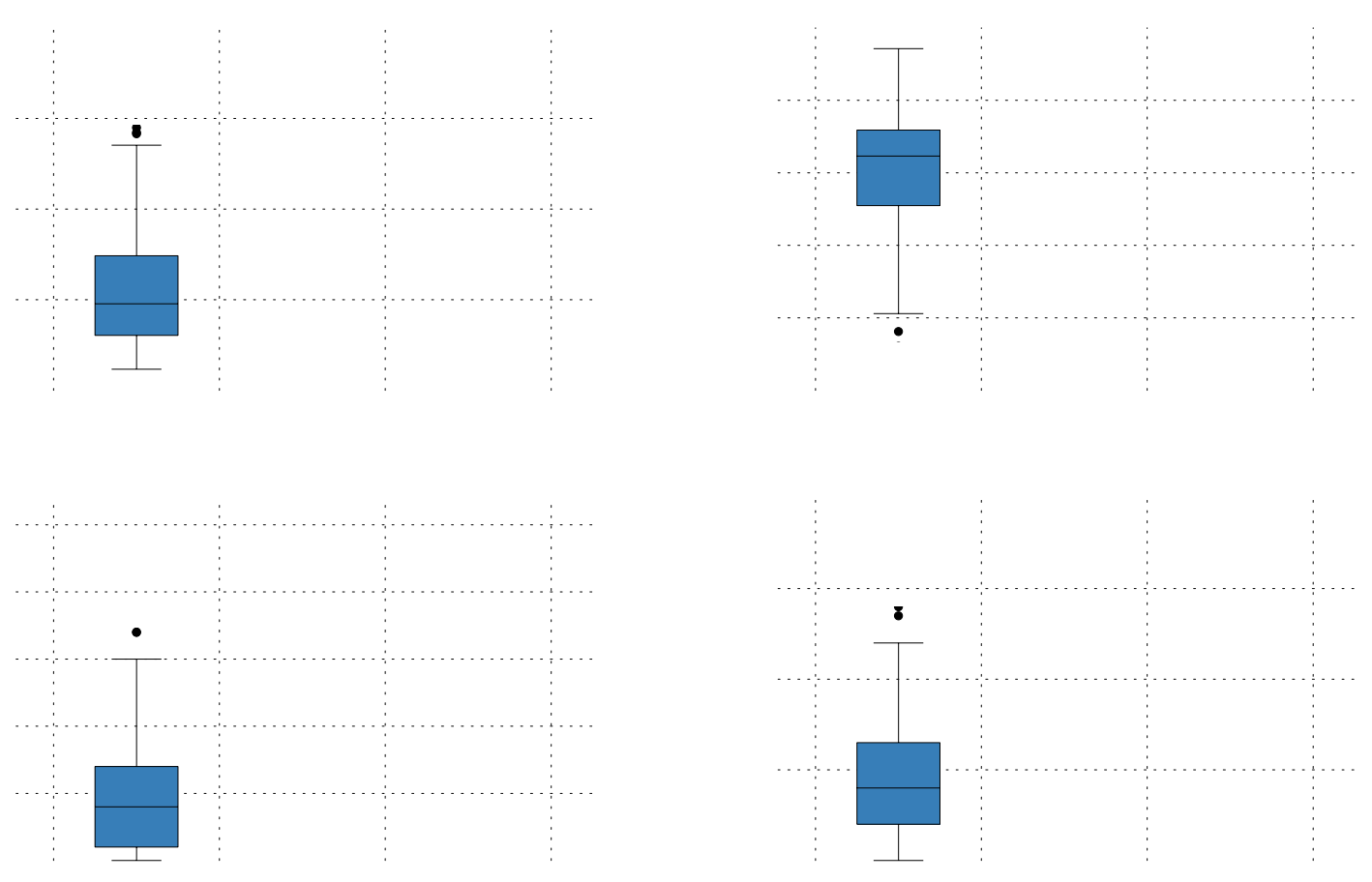
Figure 3. Ro5 property profiles for N, NB, and NI category drugs.

Studies by Wenlock et al. ${ }^{51}$ and Vieth et al. ${ }^{37}$ have shown that oral drugs have limited distributions of MW, lipophilicity, and hydrogen bonding, and generally follow Ro5 guidelines. Additionally, their overall physicochemical property profiles were reduced compared with topical, injectable, and absorbent drugs. ${ }^{37}$ The property profile of oral drugs (o) in our data set also differ markedly from that of injectables (inj) which are generally larger and more polar, and somewhat from topicals (top) which are, on average, similarly sized but slightly more lipophilic (discussed later; see also Figure S3, Supporting Information B). The “tightening up” of physicochemical properties displayed by NIcategory compounds can be attributed to two factors. First, there is greater proportion of orally administered pharmaceuticals in this group (o:inj:top = 58:23:19), for which development is guided by the Ro5, compared to N- and NB-drugs (o:inj:top = 41:37:22 for N; 51:43:6 for NB). Second, the proportion of NI-compounds was highest in two therapy areas, i.e. nervous system (44 \% of all NI compounds) and respiratory and inflammation (26\%), that consistently presented a narrow range of values across many physicochemical properties (Figure S2, Supporting Information B).

The impact of time on the physicochemical properties for the different drug categories was also examined. In the case of unmodified natural product drugs, there was a noticeable surge in the mean values for a number of properties (MW, HBA, HBD, Chiral, RTB, and PSA) from 1900 to the 1961 1980 interval before a decrease was observed (Figure S4, Supporting Information B). A corresponding reduction was evident for $c \log P$ and $\log \mathrm{D}_{7.4}$ over the same period. The temporary “bulges” parallel the rise and fall of natural product anti-infectives discovered during the "golden era” of antibiotic research from the 1930 s to early 1970 s, ${ }^{52}$ many of which were larger, more structurally complex, and less lipophilic (Table 1) than anything which had been developed prior to the 1950s. Other properties like Fsp3, RNG, and \%PSA have risen slightly and then remained relatively flat while AROM decreased between the second and third bins but has otherwise barely changed. 
Many NB-category drug properties (MW, HBD, HBA, Chiral, RTB, Fsp3, RNG, and PSA) started from a low base but increased steadily from 1900 - 2013, and eventually surpassed the mean values of unmodified natural product therapeutics by the last interval. In contrast, the lipophilic descriptors and AROM remained relatively constant. This, combined with the larger number of NB-type drugs in the data set, would contribute to the statistically insignificant changes observed for cLogP and aromatic ring count (both carbo- and hetero-) over time. Drugs inspired by a natural prototype have, for the most part, trended roughly the same as NB-category therapeutics but with lower values.

Walters and co-workers compared physicochemical properties of 1219 drugs launched from 1959 2009 with over 415,000 structures published in the Journal of Medicinal Chemistry with a MW < 1000 Da over the same time frame. ${ }^{53}$ This is the only study that has compared the properties of all drugs (i.e., the focus was not confined to oral bioavailability) with the types of compounds reported by medicinal chemists (not just those in clinical trials) over an extended period. Although there would be an obvious overlap between our data set and that of Walters et al., a general comparison between the two would nevertheless afford some insights into the similarities and differences between drugs of natural origin and their synthetic counterparts over roughly the same 50-year period.

For many of the physicochemical properties (MW, HBD, HBA, Fsp3, RTB, and PSA) tracked by Walters and colleagues in common with our investigation, the mean values of N- and NB-category compounds were found to be consistently higher than the corresponding set of drugs. For example, a difference of 300 Da in MW, 5 HBD, 8 HBA, 0.2 Fsp3, 6 RTB, and 160 $\AA^{2}$ in PSA, was observed between mean values for drugs and unmodified natural product therapeutics in the 1961 1980 reporting period. At the same time, the mean cLogP for $\mathrm{N}$-type compounds was 3 units below that of the drug set. Although this period was the most extreme for $\mathrm{N}$-compounds, the other intervals were also consistently higher. The comparisons indicate that the set of unmodified natural product drugs launched from 1960 - 2009 are, on average, larger, more flexible, more polar, more 3- 
dimensional, and have less aromatic rings than the corresponding drug set. These findings are consistent with that of Muigg et al. who used ChemGPS-NP to compare sets of natural products from marine and terrestrial sources with synthetic compounds. ${ }^{54}$

Therapeutics based on a natural prototype, although generally more moderate in terms of physicochemical properties than the N-category drugs, nevertheless followed a similar trend for all parameters, except RTB where the two sets were similar. The set of NI-type therapeutics tracked the drug set most closely, albeit with slightly higher values, for the most part.

Influence of the Therapy Area on Physicochemical Properties. The therapy area can greatly influence the physicochemical properties of drugs. ${ }^{19}$ Mean and median values of 13 physicochemical properties for the set of unique leads and drugs appear in Table 1. It is immediately obvious that the physicochemical properties presented here are markedly different to those reported by Leeson and Davis (typically higher scores in all properties, except cLogP and \%PSA which could be appreciably lower, across all therapy areas). ${ }^{19}$ There are several reasons for this. First, Leeson and Davis considered only oral drugs while this investigation also took into account non-oral routes of administration (RoA).

Vieth and co-workers have previously shown that the overall physicochemical property profiles of oral therapeutics are reduced compared with other RoA. ${ }^{37}$ Second, the present study focuses only on pharmaceuticals with a natural product pedigree. On average, natural products have higher MWs, and contain more oxygen atoms, but less nitrogen, halogen and sulfur atoms than synthetic compounds. ${ }^{55,56}$ Statistically, they are also larger, more rigid, and more stereochemically complex. ${ }^{55-57}$ Third, the time frame of Leeson and Davis’s work ranged from 1983 - 2002 (20 years), whereas this investigation captured the earliest marketed drugs prior to 1900 and extended over 115 years to 2013. Many large and complex molecules discovered during the golden age of antibiotic research, for example, would not have been published in the annual “To Market, To Market” reports used by Leeson and Davis, even if they were orally available, given these accounts commenced in 1983. Additionally, an analysis of drugs 
with a natural progenitor launched from 2001 - 2013 (as identified in this investigation) provides

evidence that there has been an increase in all physicochemical properties examined by Leeson and

Davis, except \%PSA, over the previous interval (1981 - 2000). Although this does not include

synthetic compounds over the same period, it does demonstrate how the later data have contributed to

an increase in many property profiles shown in Table 1.

Table 1. Mean (Median) Physicochemical Properties by Therapy Area of Natural Leads and

\section{Associated Drugs ${ }^{a}$}

\begin{tabular}{|c|c|c|c|c|c|c|c|}
\hline & cancer & infection & cardiovascular & $\begin{array}{l}\text { gastrointestinal } \\
\text { and metabolism }\end{array}$ & $\begin{array}{l}\text { respiratory and } \\
\text { inflammation }\end{array}$ & nervous system & others \\
\hline Lead & $n=40$ & $n=73$ & $n=36$ & $n=18$ & $n=20$ & $n=27$ & $n=10$ \\
\hline Drug & $n=59$ & $n=218$ & $n=96$ & $n=30$ & $n=82$ & $n=168$ & $n=11$ \\
\hline \multirow{2}{*}{ MW } & 598.65 (534.11) & 641.69 (500.63) & 486.01 (389.47) & 397.52 (315.95) & $509.96(314.36)$ & 309.53 (299.37) & $300.93(257.27)$ \\
\hline & $620.05(565.80)$ & $544.40(468.92)$ & $461.64(417.02)$ & 424.20 (399.55) & $392.79(296.64)$ & 343.53 (318.39) & 305.35 (296.36) \\
\hline \multirow{2}{*}{ cLogP } & $1.27(1.83)$ & $-1.93(-2.30)$ & $1.42(1.71)$ & $0.82(0.94)$ & $0.53(1.11)$ & $1.16(1.32)$ & $0.96(1.43)$ \\
\hline & $1.76(2.07)$ & $-0.98(-0.87)$ & 2.26 (2.39) & 1.54 (2.54) & $2.48(3.07)$ & 1.89 (2.37) & $1.41(1.78)$ \\
\hline \multirow{2}{*}{$\log D_{7.4}$} & $0.83(1.42)$ & $-4.45(-3.35)$ & $0.44(0.84)$ & $-0.29(-0.09)$ & $-0.80(-0.12)$ & $0.56(0.43)$ & $0.56(0.75)$ \\
\hline & 1.05 (1.14) & $-3.22(-2.90)$ & $0.87(1.02)$ & $-0.88(0.74)$ & $0.48(0.40)$ & $0.68(0.89)$ & $0.70(0.95)$ \\
\hline \multirow{2}{*}{$\operatorname{LogS}_{7.4}$} & $-3.61(-3.83)$ & $-1.19(-0.94)$ & $-2.37(-2.81)$ & $-2.34(-2.75)$ & $-2.34(-1.50)$ & $-1.70(-1.13)$ & $-2.34(-2.40)$ \\
\hline & $-3.67(-3.60)$ & $-1.93(-1.85)$ & $-2.49(-2.71)$ & $-1.82(-0.57)$ & $-1.79(-0.82)$ & $-1.21(-0.79)$ & $-2.29(-2.53)$ \\
\hline \multirow{2}{*}{ HBD } & $4.30(4.00)$ & $7.37(6.00)$ & $3.56(2.50)$ & $4.39(2.00)$ & $4.60(3.00)$ & $1.37(1.00)$ & $2.60(2.50)$ \\
\hline & $4.39(4.00)$ & $4.76(3.00)$ & $2.28(2.00)$ & $3.43(2.00)$ & $2.34(2.00)$ & $0.95(1.00)$ & $2.36(2.00)$ \\
\hline \multirow{2}{*}{ HBA } & $9.65(8.00)$ & 11.93 (12.00) & $7.64(4.50)$ & $6.50(4.00)$ & $8.50(7.00)$ & $3.56(3.00)$ & $4.40(3.50)$ \\
\hline & $9.75(9.00)$ & $9.45(8.00)$ & $6.14(5.00)$ & $6.43(5.00)$ & $5.40(4.00)$ & $3.30(3.00)$ & $4.09(3.00)$ \\
\hline \multirow{2}{*}{ Fsp3 } & $0.59(0.61)$ & $0.69(0.66)$ & $0.61(0.61)$ & $0.57(0.52)$ & $0.61(0.59)$ & $0.49(0.53)$ & $0.36(0.23)$ \\
\hline & $0.57(0.56)$ & $0.55(0.50)$ & $0.55(0.53)$ & $0.56(0.48)$ & $0.35(0.28)$ & $0.51(0.53)$ & $0.37(0.24)$ \\
\hline \multirow{2}{*}{ Chiral } & $8.48(6.50)$ & $10.37(10.00)$ & $8.64(6.50)$ & $4.67(3.50)$ & $6.80(4.00)$ & $2.81(2.00)$ & $2.20(0.00)$ \\
\hline & $7.56(6.00)$ & $6.47(4.00)$ & $5.47(3.00)$ & $3.10(1.50)$ & $2.72(1.00)$ & $2.16(2.00)$ & $1.91(0.00)$ \\
\hline \multirow{2}{*}{ RTB } & $7.58(4.50)$ & $9.51(7.00)$ & $6.28(5.00)$ & $6.83(3.00)$ & $9.25(6.00)$ & 3.19 (3.00) & $4.40(3.00)$ \\
\hline & $9.25(5.00)$ & $7.86(7.00)$ & $7.73(8.00)$ & $8.03(5.00)$ & $5.72(4.00)$ & $5.83(5.00)$ & $5.00(3.00)$ \\
\hline \multirow{2}{*}{ RNG } & $4.70(4.00)$ & $3.41(3.00)$ & $4.53(4.50)$ & $2.56(2.50)$ & $2.40(2.00)$ & $3.52(3.00)$ & $2.70(3.00)$ \\
\hline & $4.90(5.00)$ & $3.57(3.00)$ & $3.85(3.00)$ & $2.67(3.00)$ & $2.55(2.00)$ & $3.23(3.00)$ & $2.73(3.00)$ \\
\hline \multirow{2}{*}{ AROM } & $1.43(1.00)$ & $0.75(0.00)$ & $1.11(1.00)$ & $1.17(0.50)$ & $0.80(1.00)$ & $1.44(1.00)$ & $1.80(2.00)$ \\
\hline & $1.73(2.00)$ & $1.09(1.00)$ & $1.63(2.00)$ & $1.47(1.50)$ & $1.71(2.00)$ & $1.54(1.00)$ & $1.82(2.00)$ \\
\hline \multirow{2}{*}{ PSA } & 173.23 (155.29) & 237.08 (199.73) & $131.31(90.60)$ & 127.46 (71.95) & $160.80(130.35)$ & 58.91 (52.93) & 89.14 (73.06) \\
\hline & 175.48 & 182.95 & 105.87 (95.94) & $123.91(90.82)$ & 99.29 (68.92) & 53.02 (49.33) & 83.29 (68.36) \\
\hline \multirow{2}{*}{$\%$ PSA } & 23.12 (19.18) & 28.06 (27.69) & 17.79 (16.29) & 20.66 (16.70) & 20.53 (18.20) & 13.56 (11.27) & 21.07 (21.16) \\
\hline & 21.96 (18.77) & $25.80(25.33)$ & $15.26(14.73)$ & $20.08(16.01)$ & $17.67(14.91)$ & $10.23(9.25)$ & $19.05(18.06)$ \\
\hline
\end{tabular}

${ }^{a}$ Top line in each cell are mean (median) values of the lead; bottom line in each cell are the mean (median) values of the corresponding therapeutics. Various disease indications according to therapy area are listed in Table S2, Supporting Information B. 
A one-way ANOVA at a 95\% level of confidence showed that there were significant differences between the separate therapy areas for each of the physicochemical properties. While an ANOVA model is able to determine at least one significant difference between the means of various groups, it cannot identify the population means that differ substantially from each other. A post hoc analysis employing Scheffé's method ${ }^{58}$ was subsequently undertaken to determine specific physicochemical property variations across the therapy areas. Two means were accepted as significantly different if the $p$-value was $<0.05$ (Table S3, Supporting Information B). At this threshold, there was a considerable variation between therapy areas; a total of 83 of the 195 possible pairwise comparisons were found to be different (Table 2). However, it should be noted that 10 additional pairwise comparisons had a $p$-value between 0.05 and 0.1 (marked with an * in Table 2) suggesting these contrasts may also be different in a meaningful way. The large number of contrasts is probably a consequence of the relatively small numbers of drugs in some areas, and limited structural diversity in others. Anti-infective agents had the most differences (37) and, as alluded in Table 1, these were in lipophilicity and PSA. Cancer and nervous system drugs had the next highest number of contrasts (33 and 32, respectively). For anticancer therapeutics, the main physicochemical property differences were found in MW, polarity and RNG, while nervous system drugs differed from other classes mainly in MW and polarity as indicated by PSA, \%PSA, HBD, and HBA.

Interestingly, the Fsp3 profile for respiratory and inflammation was a point of contrast with all other therapy areas. Structurally, drugs for this therapy area are flatter and, except for anticancer agents, had the highest aromatic ring count, which is a complete reversal from the corresponding set of leads. This is somewhat intriguing as replacement of benzenoid rings in particular with substructures high in $\mathrm{sp}^{3}$ hybridized carbon atoms is a strategy that is currently employed to improve the properties of a candidate drug. ${ }^{15}$ 
Table 2. Scheffé's Test Analysis of Physicochemical Properties for Drugs Developed from a

Natural Prototype in the Six Major Therapy Areas ${ }^{a}$

\begin{tabular}{|c|c|c|c|c|c|}
\hline & cancer & infection & $\mathrm{CV}$ & GI \& M & R \& I \\
\hline infection & $\begin{array}{l}\text { cLogP, } \log \text {, } \\
\text { \%PSA*, RNG, } \\
\text { AROM, LogS }\end{array}$ & & & $\log D^{*}$ & \\
\hline $\mathrm{CV}$ & $\begin{array}{lr}\text { MW, } & \text { HBD, } \\
\text { HBA, } & \text { PSA, } \\
\text { \%PSA, } & \text { RNG, } \\
\text { LogS* } & \end{array}$ & $\begin{array}{l}\text { cLogP, LogD, } \\
\text { HBD, HBA, } \\
\text { PSA, \%PSA, } \\
\text { AROM }\end{array}$ & & & \\
\hline GI \& M & $\begin{array}{l}\text { MW, } \mathrm{HBA}^{*} \text {, } \\
\text { Chiral, } \mathrm{RNG} \text {, } \\
\text { LogS }\end{array}$ & $\begin{array}{l}\text { cLogP, LogD*, } \\
\text { HBA, PSA, } \\
\text { \%PSA, Chiral }\end{array}$ & $\mathrm{RNG}^{*}$ & & \\
\hline R \& I & $\begin{array}{ll}\text { MW, } & \text { HBD, } \\
\text { HBA, } & \text { PSA, } \\
\text { \%PSA*, } & \\
\text { Chiral, } & \text { RTB, } \\
\text { RNG, } & \text { Fsp3, } \\
\text { LogS } & \end{array}$ & $\begin{array}{l}\text { MW, } \\
\text { LogD, } \\
\text { HBA, } \\
\text { HBA, } \\
\text { \%PSA, Chiral, } \\
\text { RNG, AROM, } \\
\text { Fsp3 }\end{array}$ & $\begin{array}{l}\text { Chiral, RNG, } \\
\text { Fsp3 }\end{array}$ & Fsp3 & \\
\hline nervous system & $\begin{array}{lr}\text { MW, } & \text { HBD, } \\
\text { HBA, } & \text { PSA, } \\
\text { \%PSA, } & \text { Chiral, } \\
\text { RTB, } & \text { RNG, } \\
\text { LogS } & \end{array}$ & $\begin{array}{l}\text { MW, } \\
\text { LogD, } \\
\text { HBA, } \\
\text { \%PSA, } \\
\text { RTA } \\
\text { RTA, } \\
\text { LogS* }\end{array}$ & $\begin{array}{l}\text { MW, } \\
\text { HBA, } \\
\text { \%PSA, } \\
\text { LogA, } \\
\text { LogS }\end{array}$ & $\begin{array}{l}\text { HBD, HBA, } \\
\text { PSA, \%PSA }\end{array}$ & $\begin{array}{l}\mathrm{HBD}^{*}, \mathrm{HBA}, \\
\text { PSA, \%PSA, } \\
\text { Fsp3 }\end{array}$ \\
\hline
\end{tabular}

\footnotetext{
${ }^{a} \mathrm{CV}$, cardiovascular; GI \& M, gastrointestinal and metabolism; R \& I, respiratory and inflammation;
} the $p$-value for the pairwise comparisons denoted with an * was in the range $0.05-0.1$.

Of all the therapy areas, cancer contained the highest percentage of N-type drugs (42\%, Figure 4), and the largest amount of prototypes relative to drug products (Table 1). The disproportionately high number of therapeutics in the $\mathrm{N}$ category (25) helped minimize differences between lead and drug sets relative to other areas for mean and median structure-related properties (e.g. MW, HBD, HBA). The mean MW of lead molecules for cancer was the second highest of all therapy areas and the median was highest. However, unlike other areas where the MW decreased for the corresponding set of drugs when the mean MW of the lead compounds was 500 Da, anticancer agents increased. This trend is apparent 
in four prototypes (camptothecin, doxorubicin, podophyllotoxin, and spongothymidine) that afforded at least three drugs each, effectively accounting for half of all NB and NI drugs with a higher MW than the progenitor.
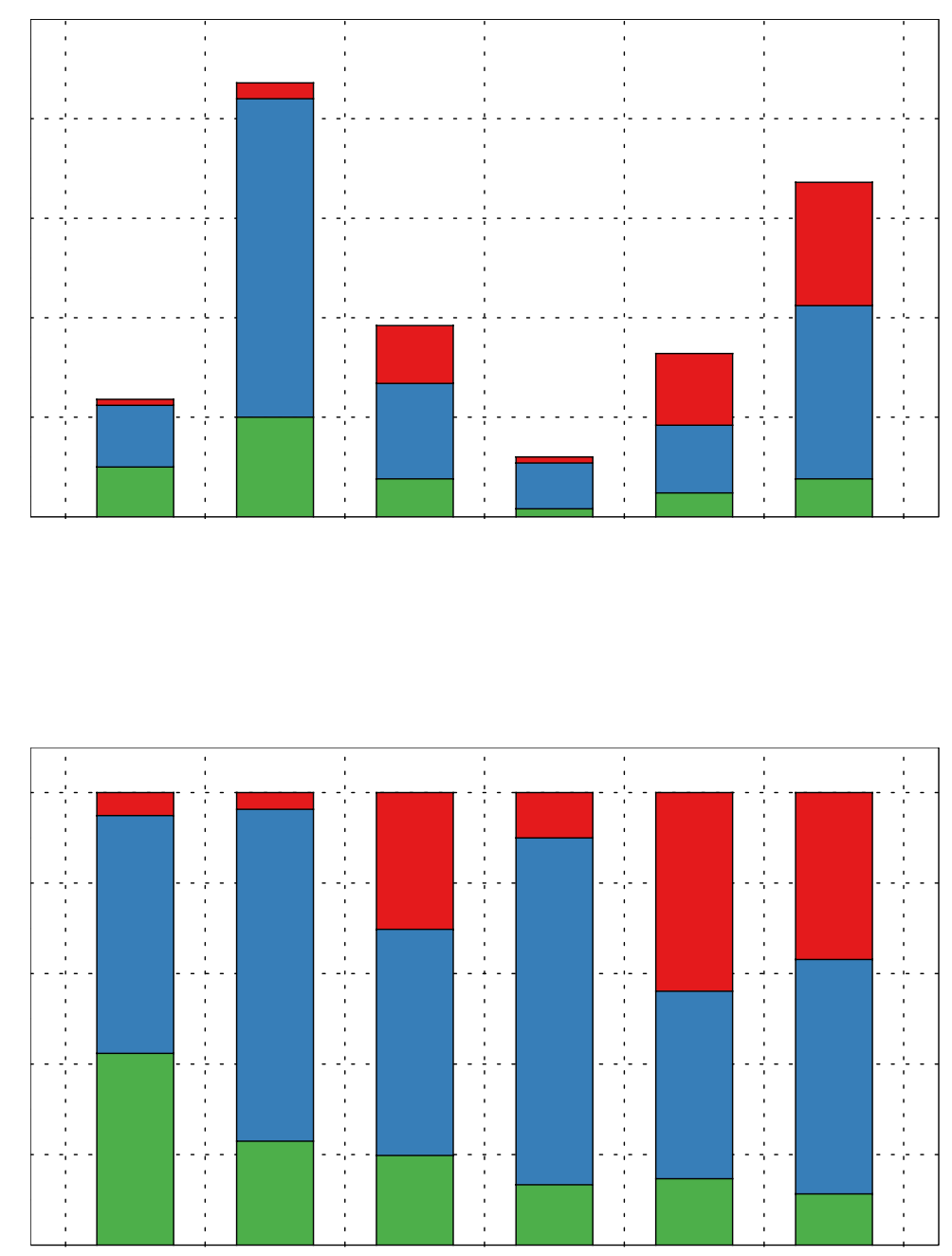

Figure 4. Contribution of N, NB, and NI category drugs to each of the major therapy areas by count and percentage (CV, cardiovascular; GI\&M, gastrointestinal and metabolism; R\&I, respiratory and inflammation).

Statistically, anti-infective prototypes were significantly more polar than progenitors from other therapy areas as reflected by their higher mean PSA and \%PSA values, and lowest mean lipophilicity (Table 1). 
They were also the most structurally complex, having the uppermost mean scores for MW, HBD, HBA, Fsp3, Chiral, and RTB. Although the drugs developed from anti-infective prototypes were typically smaller and less complex than the lead compound, and tended to fall within Lipinski guidelines, they were nevertheless larger and more complex than therapeutics from almost every other therapy area except cancer. These combined observations suggest that, to be effective in a nonhuman organism, antiinfectives may require a more multifaceted topology with an increased number of polar functional groups arranged strategically in 3D space to facilitate penetration through xenobiotic cell walls if an active transport mechanism is not operating. As shown in Figure 4, the anti-infective therapy area had a significantly higher percentage of drugs in the NB category (73\%) compared with N (23\%) and NI (4\%, eight compounds). Two leads, penicillin G (6) and cephalosporin C, accounted for approximately 55\% of all NB and NI drugs. Importantly, another eight natural leads were developed into four or more therapeutics so that, in essence, 10 prototypes afforded over $80 \%$ of all NB and NI anti-infective drugs.

In contrast, both nervous system leads and drugs are significantly smaller in terms of MW. They were also less polar as adjudged by the lower HBD and HBA count, and smaller values for PSA and \%PSA (Figure 5). Nervous system leads are structurally less complex compared with other therapy areas as shown by the lower mean scores for Fsp3, Chiral, and RTB. The corresponding drug data for mean Fsp3, Chiral, and RTB indicates that lead optimization results in a set that has a decreased number of stereogenic atoms coupled with a small amount of additional flexibility. For compounds acting on the central nervous system, which the majority of these are, the physicochemical properties presented in Table 1 are consistent with the recognized importance of employing lipid-soluble small molecules with MW $<400$ Da to facilitate crossing of the blood-brain barrier. ${ }^{59,60}$ The combined NB and NI categories accounted for $89 \%$ of all therapeutics in the nervous system therapy area. The majority of drugs $(\sim 80 \%)$ from these two classifications were represented by six prototypes that afforded a minimum of six drugs each (i.e. atropine, cocaine, morphine, ergometrine, tubocurarine, and salicin). 
The remaining therapy areas (cardiovascular, gastrointestinal and metabolism, and respiratory and inflammation) have physicochemical property profiles for the drug sets that fall within the Ro5 (Figures 5 for Ro5 properties, PSA, and \%PSA, and S2, Supporting Information B, for all 13 physicochemical properties). Interestingly, all but one NI drug in the respiratory and inflammation therapy area can be traced back to salicin suggesting the chemical space for new leads from natural sources may be rather limited, or under-explored, if only small molecules are being considered.
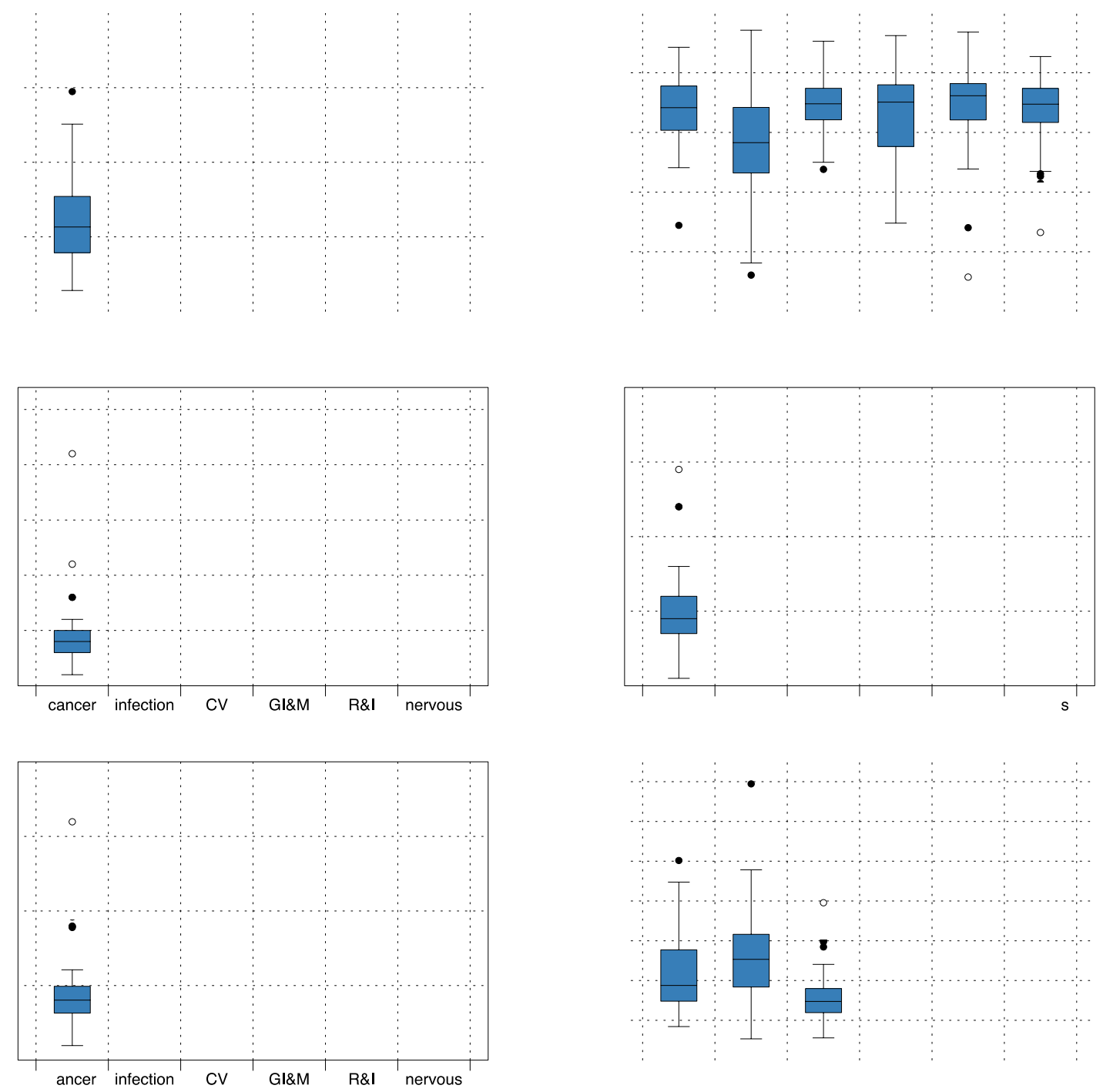

Figure 5. Distribution of Ro5, PSA and \%PSA properties for drugs in each of the major therapy areas (CV, cardiovascular; GI\&M, gastrointestinal and metabolism; R\&I, respiratory and inflammation). 
If anti-infective agents were excluded, the remaining therapeutics occupied a well-defined range for cLogP $(1.50-2.50)$. A one-way ANOVA of the abridged set at a 95\% confidence level indicated that only cLogP and AROM were statistically similar as shown by $p=0.29$ and 0.57 , respectively. The fact that these two parameters do not change in a statistically meaningful way across five of the six therapy areas emphasizes their importance in natural product drug discovery considering they are also independent of the year of launch.

Influence of the Route of Administration on Physicochemical Properties. Finally, we also examined the RoA of drugs developed from a natural prototype to determine whether or not there may be an association with physicochemical properties (Figure S3, Supporting Information B). Vieth and co-workers had previously analyzed the physicochemical properties of 1729 marketed drugs. ${ }^{37}$ The authors approached the statistical analysis by first prioritizing the RoA in the order: oral > topical > absorbent > injectable. Thus, if a drug had two or more RoA, then it was counted only once in the route of highest priority. From a list of 1729 marketed drugs, 1193 were consequently assigned as oral; 112 as topical; 116 as absorbent, and the remaining 308 as injectables. ${ }^{37}$

Our approach differed in three ways. First, we noted no drugs in this set were defined as absorbents by the FDA’s Approved Drug Products with Therapeutic Equivalence Evaluations (Orange Book) ${ }^{61}$ Second, a new category, inhalants, was added to capture those drugs administered via aerosolization. Third, all instances of the RoA were counted. This last point of difference was done for two reasons: 1) a greater understanding of each RoA area could potentially be gained if all drugs administered via a particular route were counted, and 2) we hypothesized that drugs derived from natural products may not necessarily be as biased towards oral bioavailability as synthetics; however, this thesis could not be tested if instances of routes other than oral were not captured.

Before continuing, it is also worth noting that the identified classifications for RoA may be misleading for a small minority of compounds; in particular oral administration does not necessarily equate to oral 
bioavailability. By way of example, the lipoglycopeptide antibacterial, teicoplanin A $_{2}-3$ (from the 1961 - 1980 bin), violates three Ro5 physicochemical properties, i.e. MW 1880 Da, HBD = 24, HBA = 31, and two of these parameters (MW and HBA) are extreme outliers on the Tukey plots (Figures 2 and 5). However, even though teicoplanin is administered orally for the treatment of antibiotic-associated colitis, ${ }^{40}$ it is nevertheless poorly absorbed by this route compared with $90 \%$ bioavailability via injection. ${ }^{62}$

Using the Orange Book, Martindale The Complete Drug Reference ${ }^{40}$ and other resources (Experimental Section), data was obtained for 647 (97.5\%) of the therapeutics. The vast majority (630, 95\%) could be assigned to one of eight possible combinations: oral (o); injectable (inj); topical (top); o + inj; o + top; o + inj + top; inj + top; inhalant (inh) (Table 3). The inter-relationship between the three major RoA areas that, combined, account for $\sim 94 \%$ of all data is shown in Figure 6.

Table 3. Route of Administration Combinations for Drugs Developed from a Natural Lead

\begin{tabular}{lllllll}
\hline RoA Comb. $^{a}$ & $n^{b}$ & \% Contribution & Cumulative \% & $\mathrm{N}$ & $\mathrm{NB}$ & $\mathrm{NI}$ \\
\hline o & 252 & 38 & 38 & 31 & 152 & 69 \\
inj & 172 & 26 & 64 & 31 & 120 & 21 \\
o + inj & 99 & 15 & 79 & 24 & 63 & 12 \\
top & 33 & 5 & 84 & 15 & 7 & 11 \\
o + top & 35 & 5 & 89 & 11 & 8 & 16 \\
o + inj + top & 27 & 4 & 93 & 13 & 11 & 3 \\
inj + top & 8 & 1.25 & 94.25 & 3 & 2 & 3 \\
inh & 5 & 0.75 & 95 & 0 & 5 & 0 \\
others & 16 & 2.5 & 97.5 & 4 & 10 & 3 \\
unknown & 17 & 2.5 & 100 & 5 & 8 & 4 \\
\hline
\end{tabular}


${ }^{a}$ RoA Comb., route of administration combinations; ${ }^{b}$ Total $n=664 ;{ }^{c}$ eight other groups, each with $<$ 5 entries.

While direct comparisons cannot be made between the analysis undertaken by Vieth et al. and here due to the different methodologies, Figure 6 suggests that the proportion of injectables is significant for the set of drugs developed from a natural product.

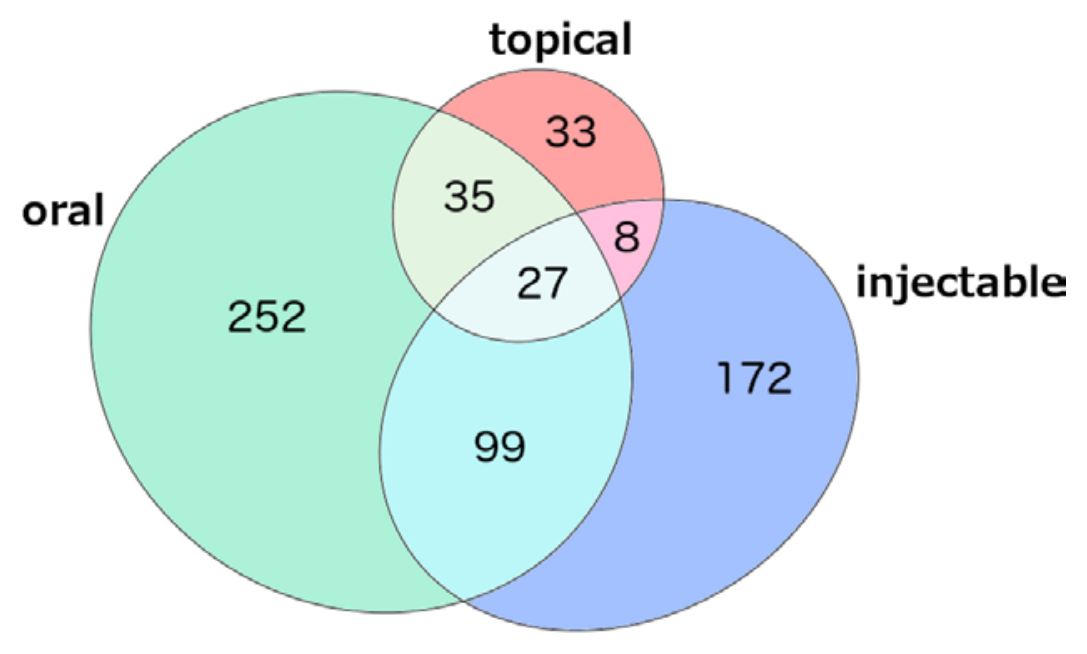

Figure 6. Distribution of the three major RoA areas for therapeutics developed from a natural prototype.

The mean and median values of the 13 physicochemical properties presented in Table 4 indicate that injectables would be significantly different to the other two major RoA areas, especially in lipophilicity. A one-way ANOVA at a 95\% level of confidence confirmed variation between the three major RoA for most physicochemical properties, and a post hoc analysis using Scheffé's method ${ }^{58}$ revealed that injectables contributed to all 20 contrasts of the 39 possible pairwise comparisons where the $p$-value < 0.05 (Table 5 below, and Table S4, Supporting Information B), clearly demonstrating the greatest overall differences in physicochemical properties from the other RoA areas.

Interestingly, ANOVA indicated that there was no significant difference in solubility (as defined by $\operatorname{LogS}_{7.4}$ ) between the three groups, suggesting that the development of natural prototypes into drugs 
(other than inhalants) may all follow a similar strategy that ultimately results in therapeutics with similar solubility profiles. The data in Table 4 indicates that the mean calculated values of $\operatorname{LogS}_{7.4}$ for the lead compounds in the oral, injectable, and topical routes was higher in all cases than the corresponding set of drugs. This may be explained by an increase in AROM (and concomitant decrease in Fsp3) which, in turn affects lipophilicity. Presumably, these modifications were part of a general optimization strategy that sought to increase potency.

Table 4. Mean (Median) Physicochemical Properties by Route of Administration Area of Natural Leads and Associated Drugs ${ }^{a}$

\begin{tabular}{lllll}
\hline & total oral & total injectable & total topical & total inhalants \\
$\begin{array}{l}\text { Lead:Drug } \\
\text { Pairs }\end{array}$ & $n=421$ & $n=313$ & $n=110$ & $n=18$ \\
\hline MW & $408.57(320.34)$ & $474.13(389.55)$ & $398.47(302.37)$ & $290.12(285.81)$ \\
& $415.62(360.47)$ & $519.69(444.44)$ & $412.43(323.13)$ & $364.87(353.92)$ \\
cLogP & $0.34(1.04)$ & $-0.47(0.90)$ & $0.56(0.90)$ & $0.73(1.24)$ \\
& $1.72(2.28)$ & $0.02(0.73)$ & $1.73(2.50)$ & $0.45(1.32)$ \\
LogD $_{7.4}$ & $-0.89(-0.60)$ & $-2.17(-0.60)$ & $-0.60(-0.41)$ & $-0.46(-0.41)$ \\
& $0.22(0.57)$ & $-1.72(-0.79)$ & $0.02(0.90)$ & $-2.09(-1.79)$ \\
LogS $_{7.4}$ & $-1.53(-0.94)$ & $-1.42(-0.69)$ & $-1.54(-0.94)$ & $-1.10(-0.08)$ \\
& $-2.04(-1.86)$ & $-1.80(-1.48)$ & $-1.86(-1.45)$ & $-0.64(0.00)$ \\
HBD & $3.40(2.00)$ & $4.12(3.00)$ & $3.37(2.00)$ & $1.89(1.00)$ \\
& $2.42(2.00)$ & $3.82(3.00)$ & $2.78(1.00)$ & $2.33(2.00)$ \\
HBA & $6.44(4.00)$ & $7.68(6.00)$ & $6.45(5.00)$ & $4.39(4.00)$ \\
& $5.68(5.00)$ & $8.06(7.00)$ & $5.85(4.00)$ & $5.39(3.00)$ \\
Fsp3 & $0.54(0.54)$ & $0.56(0.53)$ & $0.56(0.54)$ & $0.50(0.53)$ \\
& $0.49(0.49)$ & $0.55(0.52)$ & $0.51(0.53)$ & $0.47(0.46)$ \\
Chiral & $5.58(5.00)$ & $6.09(4.00)$ & $5.85(5.00)$ & $3.61(4.00)$ \\
& $4.20(3.00)$ & $5.62(4.00)$ & $4.48(2.00)$ & $2.89(2.00)$ \\
& & & &
\end{tabular}




$\begin{array}{lllll} & 5.30(4.00) & 6.30(5.00) & 5.19(4.00) & 3.17(3.50) \\ \text { RTB } & 6.00(5.00) & 8.01(6.00) & 6.25(5.00) & 5.28(6.00) \\ & 3.44(3.00) & 3.85(3.00) & 3.02(3.00) & 3.17(3.00) \\ \text { AROM } & 3.30(3.00) & 3.95(4.00) & 2.86(3.00) & 3.44(3.00) \\ & 1.16(1.00) & 1.19(1.00) & 0.99(1.00) & 1.39(1.00) \\ \text { PSA } & 1.46(1.00) & 1.46(1.00) & 1.20(1.00) & 1.83(1.50) \\ & 120.23(86.71) & 148.28(115.89) & 117.40(89.34) & 73.92(57.90) \\ & 102.21(80.56) & 150.66(126.44) & 107.29(64.79) & 94.15(59.18) \\ & 20.68(19.18) & 22.60(19.18) & 20.70(16.35) & 17.43(14.04) \\ & 17.02(14.73) & 20.40(18.76) & 17.45(13.99) & 17.63(13.13)\end{array}$

${ }^{a}$ Top line in each cell are mean (median) values of $n$ leads for each route of administration; bottom line in each cell are the mean (median) values of the corresponding $n$ therapeutics; ${ }^{b}$ All instances of oral, injectable, topical, and inhalant drugs were counted.

Table 5. Scheffé's Test Analysis of Physicochemical Properties for Drugs Developed from a Natural Prototype for the Three Major Routes of Administration ${ }^{a}$

\begin{tabular}{lll}
\hline & oral & injectable \\
\hline injectable & MW, cLogP, LogD, HBD, & \\
& HBA, PSA, \%PSA, & \\
& Chiral, RTB, RNG, Fsp3 & \\
topical & RNG*, AROM* & MW, cLogP, LogD, HBD, \\
& & HBA, PSA, \%PSA, RTB, \\
& & RNG, AROM* \\
\hline
\end{tabular}

${ }^{a}$ The $p$-value for the pairwise comparisons denoted with an * was in the range $0.05-0.1$.

Natural progenitors and the drugs developed from them that were ultimately formulated as injectables had significantly higher MW, were more structurally complex, and less lipophilic than other RoA areas. This combination was reminiscent of trends found in anti-infective agents, alluding that this therapy area may be a significant contributor. This was confirmed when the set of 313 injectables was crossreferenced against the therapy areas. Anti-infective therapeutics accounted for $40 \%$ of the entries, 
approximately a quarter were nervous system drugs, and anticancer agents contributed 17\% (Table 6). Significantly, 53 anticancer drugs were formulated as injectables; this is a $90 \%$ contribution from this therapy area, compared to 124 anti-infectives (57\% of the therapy area), and 73 nervous system therapeutics (43\% of the therapy area). Cancer therapeutics contributed $1 \%$ to orals and $4 \%$ to topical applications. High contributions to a specific RoA were also observed in the case of orally administered drugs for the cardiovascular, and gastrointestinal and metabolism therapy areas (90 and 97\%, respectively).

Table 6. Percent Contributions of Therapy Areas to the Three Major Routes of Administration ${ }^{a}$

\begin{tabular}{|c|c|c|c|c|c|c|c|c|c|}
\hline & \multicolumn{3}{|l|}{ oral } & \multicolumn{3}{|c|}{ injectable } & \multicolumn{3}{|c|}{ topical } \\
\hline & $n$ & $\% \operatorname{RoA}^{b}$ & $\% \mathrm{TA}^{c}$ & $n$ & \% RoA & $\%$ TA & $n$ & \% RoA & $\%$ TA \\
\hline cancer & 6 & 1 & 10 & 53 & 17 & 90 & 4 & 4 & 7 \\
\hline infection & 115 & 28 & 53 & 124 & 40 & 57 & 34 & 31 & 26 \\
\hline $\mathrm{CV}^{d}$ & 86 & 21 & 90 & 37 & 12 & 39 & 4 & 4 & 41 \\
\hline GI\&M ${ }^{e}$ & 29 & 7 & 97 & 2 & 1 & 7 & 2 & 2 & 7 \\
\hline $\mathrm{R} \& \mathrm{I}^{f}$ & 63 & 15 & 77 & 18 & 6 & 22 & 24 & 24 & 27 \\
\hline $\begin{array}{l}\text { nervous } \\
\text { system }\end{array}$ & 114 & 28 & 68 & 73 & 24 & 43 & 40 & 37 & 24 \\
\hline
\end{tabular}

${ }^{a}$ All instances of oral, injectable, and topical drugs were counted, therefore total $n$ for each therapy area will not equal drug count from Table 1 when a drug has two or more routes of administration; ${ }^{b}$ RoA, route of administration; ${ }^{c} \mathrm{TA}$, therapy area; ${ }^{d} \mathrm{CV}$, cardiovascular; ${ }^{e} \mathrm{GI} \& \mathrm{M}$, gastrointestinal and metabolism; ${ }^{f}$ R\&I, respiratory and inflammation.

Although the count for drugs delivered as inhalants is low, a trend towards lower size and polarity is nevertheless apparent in both the leads and pharmaceuticals. Most of the inhalants (16) were divided evenly between the respiratory and inflammation, and nervous system therapy areas. Not unexpectedly, these therapy areas score the lowest in mean MW and structural complexity, and highest in lipophilicity (Table 1). The set of drugs from this data set returned different results compared with an analysis of the 
calculated physicochemical properties of 81 respiratory drugs delivered via the oral and inhalant routes. ${ }^{63}$ Here, 29 drugs administered exclusively by the intranasal/inhaled routes were found to have higher MW, HBD and HBA, and lower lipophilicity compared with oral drugs. Notably, the authors observed that the MW was not significantly different to oral drugs after glucocorticoids were removed indicating that, on average, the remaining inhalants were generally smaller molecules on par with orals. $^{63}$

In conclusion, the data in this report shows that the mean values of lipophilicity and aromatic ring count for drugs developed from natural prototypes have not changed significantly over the five intervals that extend from pre-1900 to 2013. Moreover, with the exception of anti-infective drugs, lipophilicity, and aromatic ring count are also independent of therapy area. This would suggest that these two properties are of primary importance when prosecuting a natural product drug discovery program, but of the two, lipophilicity reigns supreme.

A comparison of physicochemical properties for both leads and drugs by therapy area shows that antiinfective classes have a markedly different profile. Anti-infective leads were larger, more polar, more water soluble, and considerably less lipophilic than progenitors from other therapy areas. Similar profiles were observed for anti-infective therapeutics, except for MW where anticancer agents were largest. The disproportionately high number of natural leads and N-type drugs from the cancer therapy area resulted in less variation between the mean scores of the prototype and therapeutic compared with other areas. In other therapy areas, certain chemotypes were successfully exploited to afford a large number of NB- or NI-type therapeutics. By way of example, 10 of the 79 prototypes in the infective therapy area accounted for $80 \%$ of all anti-infective agents; two, penicillin G (6) and cephalosporin C contributed 55\%. One prototype, salicin dominated respiratory and inflammation, underpinning a massive $60 \%$ of all non-N compounds. In the nervous system therapy area, six prototypes delivered $\sim 80 \%$ of all therapeutics. Consistent with the previous computational study by Leeson and Davis, ${ }^{19}$ and 
empirical data reported by Gleeson, ${ }^{60}$ nervous system drugs were smaller, less structurally complex, and less polar compared with other areas. However, it is vital to note that as more biologically active novel natural products are discovered and developed into therapeutics, or older prototypes exploited in new ways, the results of the present study may change. The current analysis will not necessarily be a reliable indicator of future trends.

Previous investigations of physicochemical properties have generally been in the context of oral bioavailability which has excluded many drugs developed from a natural prototype. While it is appropriate that libraries based on natural products are analyzed for oral bioavailability in certain situations, e.g. neglected diseases where logistics and compliance considerations are extremely important, in other circumstances it may be worthwhile to consider administration via other routes, especially if their molecular properties predispose them towards formulation as injectables. Many innovative first-in-class therapeutics, typically with novel chemotypes, for the treatment of various cancers [e.g. doxorubicin and analogues, taxol (1), eribulin (8), ixabepilone (13)], or infections (e.g. caspofungin, cephamycins, and the majority of penems) were formulated as injectables.

Like Vieth et al., we also observed a pronounced difference between drugs administered via injection, and all other RoA. Particularly noticeable were the higher MW and lower cLogP ranges. We have attributed this to a high proportion of anti-infective agents, and large percentage contribution from the cancer therapy area; the general physicochemical properties of both being echoed in the injectable RoA. ANOVA between the three major RoA indicated that solubility as computed by $\operatorname{LogS}_{7.4}$ was not statistically significant. We have attributed this to the editing in of aromatic rings that increases lipophilicity, and ultimately potency, en route to the drug.

Finally, the progression of a natural prototype to a drug does not always follow the current modus operandi of starting small then adding mass and functionality to deliver a therapeutic, typically for oral administration. A not insignificant amount of natural prototypes ( 20\%) proceeded directly to the 
therapeutic with no further modification. For NB and NI category drugs, simplification of larger lead structures could also be pursued to afford a therapeutic. Given the data indicates that, on average, natural progenitors are becoming progressively larger and more structurally complex, this strategy should become more established in the context of natural product drug discovery.

\section{EXPERIMENTAL SECTION}

Data Gathering. The data were gathered with the goal of compiling the largest possible set of smallmolecule drugs based on a natural prototype, and reflective of a biodiscovery program. A series of reviews by Newman et al. ${ }^{28-31}$ and by Butler ${ }^{34-36}$ facilitated compilation of data associated with new drug approvals from 1981 onwards. Efforts to identify earlier therapeutics and their lead compounds were guided by Sneader ${ }^{39}$ and the Pharmaceutical Manufacturing Encyclopedia (PME). ${ }^{64}$ Older therapeutics that may not have been approved with the same rigor, as is the case today, were not necessarily omitted if evidence was available to support their inclusion. Here, Martindale The Complete Drug Reference (Martindale) ${ }^{40}$ was a primary resource. After applying four filters to exclude certain entries (Results and Discussion Section), a data set comprising 664 drugs was obtained. CAS numbers and INCHIKeys were used to double check the data prior to the calculations being performed.

Assignment of Therapy Area. Disease indications listed in the “To Market, To Market” sections from Annual Reports of Medicinal Chemistry, ${ }^{65}$ Appendix 2 of the Dictionary of United States Adopted Names and International Drug Names, ${ }^{66}$ the ChemIDplus database, ${ }^{67}$ and the $\mathrm{PME}^{64}$ were used to classify the 664 drugs into one of the six major therapy areas previously employed by Leeson and Davis. $^{19}$

Assignment of Route of Administration Area. Four broad routes of administration listed in the FDA’s Approved Drug Products with Therapeutic Equivalence Evaluations (Orange Book) ${ }^{61}$ were used in this study: oral, injectable, topical, and inhalant. Oral drugs also included sublingual delivery as previously explained by Vieth et al. ${ }^{37}$ Injectables encompassed the more common intravenous, intramuscular and subcutaneous routes as well as other less common routes typically employing a 
syringe and hollow needle, e.g. intrathecal and intra-arterial mechanisms. Topical formulations included creams, gels, lotions, oils, ointments, pastes, solutions, sprays, and suppositories for dermatologic, ophthalmic, otic, rectal, and vaginal administration not intended for systemic drug absorption. $^{61}$ Inhalants are comprised of solutions and powders intended for aerosolization. ${ }^{61}$ The Orange Book was employed for determining the various routes of administration for more recent drugs approved by the FDA, while Martindale ${ }^{40}$ was used primarily for older drugs, or those approved outside of the USA. Other web-based resources such as SciFinder ${ }^{68}$ and Google Books were also used to find the route of administration for older drugs that had been withdrawn from the market and for which details were often sparse.

Calculation of Physicochemical Properties and Statistical Analysis. Calculation of 13 physicochemical properties for both the set of leads and drug was performed with ChemAxon's Instant JChem version 15.1.19.0. The computed descriptors afforded a comprehensive coverage of the key bulk characteristics of each molecule. One-way ANOVA for drug physicochemical properties with respect to time, therapy area, and route of administration were carried out using IBM's SPSS. ${ }^{69}$ Post hoc analysis using Scheffé's method ${ }^{58}$ at a 95\% level of confidence were performed in SPSS using the default settings for therapy area and route of administration.

\section{ASSOCIATED CONTENT}

Supporting Information Available: PDF tables of calculated physicochemical properties for natural leads and drugs, and associated data. PDF containing additional figures of 13 physicochemical property profiles for sets of drugs, drug categories, therapy areas and routes of administration; table of change in mean (median) physicochemical properties for drugs up to, and from 1981; table of indication and major therapy areas; tables of pairwise comparisons. This material is available free of charge via the Internet at http://pubs.acs.org.

\section{AUTHOR INFORMATION}




\section{Corresponding Authors}

*E-mail: david.camp@griffith.edu.au. Phone: (+61)7 37354009.

*E-mail: marc.campitelli@qimrberghofer.edu.au. Phone: (+61)7 33620222.

\section{Notes}

The authors declare no competing financial interest.

\section{ACKNOWLEDGMENTS}

ChemAxon (http://www.chemaxon.com) is gratefully acknowledged for an academic license of Instant JChem version 15.1.19.0, 2015, for calculation of all physicochemical properties used in this manuscript. We also thank Dr Michael Vieth (Lilly Research Laboratories) for helpful comments related to analyses of drugs with multiple routes of administration. The assistance of Prof. Darryl Hawker and Mr Michael Arthur (Griffith University) for critically reading the manuscript, and consultation on the statistical data analysis, respectively, is also thankfully acknowledged.

\section{REFERENCES}

(1) Lipinski, C. A.; Lombardo, F.; Dominy, B.; Feeney, P. J. Adv. Drug Del. Rev. 1997, 23, 3-25.

(2) Hann, M. M. Med. Chem. Commun. 2011, 2, 349-355.

(3) Kell, D. B.; Dobson, P. D.; Bilsland, E.; Oliver, S. G. Drug Discov. Today 2013, 18, 218-239.

(4) Kell, D. B.; Dobson, P. D.; Oliver, S. G. Drug Discov. Today 2011, 16, 704-714.

(5) Di, L.; Artursson, P.; Avdeef, A.; Ecker, G. F.; Faller, B.; Fischer, H.; Houston, J. B.; Kansy, M.; Kerns, E. H.; Krämer, S. D.; Lennernäs, H.; Sugano, K. Drug Discov. Today 2012, 17, 905-912.

(6) Sugano, K.; Kansy, M.; Artursson, P.; Avdeef, A.; Bendels, S.; Di, L.; Ecker, G. F.; Faller, B.; Fischer, H.; Gerebtzoff, G.; Lennernaes, H.; Senner, F. Nat. Rev. Drug Discov. 2010, 9, 597-614.

(7) Macarron, R.; Luengo, J. I. Future Med. Chem. 2011, 3, 505-507.

(8) Ganesan, A. Curr. Opin. Chem. Biol. 2008, 12, 306-317. 
(9) Gleeson, M. P.; Hersey, A.; Montanari, D.; Overington, J. Nat. Rev. Drug Discov. 2011, 10, 197208.

(10) Veber, D. F.; Johnson, S. R.; Cheng, H.-Y.; Smith, B. R.; Ward, K. W.; Kopple, K. D. J. Med. Chem. 2002, 45, 2615-2623.

(11) Lu, J. J.; Crimin, K.; Goodwin, J. T.; Crivori, P.; Orrenius, C.; Xing, L.; Tandler, P. J.; Vidmar, T. J.; Amore, B. M.; Wilson, A. G. E.; Stouten, P. F. W.; Burton, P. S. J. Med. Chem. 2004, 47, 61046107.

(12) Bickerton, G. R.; Paolini, G. V.; Besnard, J.; Muresan, S.; Hopkins, A. L. Nat. Chem. 2012, 4, 90-98.

(13) Ritchie, T. J.; Macdonald, S. F. Drug Discov. Today 2009, 14, 1011-1020.

(14) Ritchie, T. J.; Macdonald, S. J. F. J. Med. Chem. 2014, 57, 7206-7215.

(15) Lovering, F.; Bikker, J.; Humblet, C. J. Med. Chem. 2009, 52, 6752-6756.

(16) Hill, A. P.; Young, R. J. Drug Discov. Today 2010, 15, 648-655.

(17) Hartmann, T.; Schmitt, J. Drug Discov. Today: Technol. 2004, 1, 431-439.

(18) Charifson, P. S.; Walters, W. P. J. Med. Chem. 2014, 57, 9701-9717.

(19) Leeson, P. D.; Davis, A. M. J. Med. Chem. 2004, 47, 6338-6348.

(20) Leeson, P. D.; St-Gallay, S. A.; Wenlock, M. C. Med. Chem. Commun. 2011, 2, 91-105.

(21) Martin, Y. C. J. Med. Chem. 2005, 48, 3164-3170.

(22) Hann, M. M.; Oprea, T. I. Curr. Opin. Chem. Biol. 2004, 8, 255-263.

(23) Oprea, T. I.; Davis, A. M.; Teague, S. J.; Leeson, P. D. J. Chem. Inf. Comp. Sc. 2001, 41, 13081315.

(24) Teague, S. J.; Davis, A. M.; Leeson, P. D.; Oprea, T. Angew. Chem. Int. Ed. Engl. 1999, 38, 3743-3747.

(25) Camp, D.; Davis, R. A.; Campitelli, M.; Ebdon, J.; Quinn, R. J. J. Nat. Prod. 2012, 75, 72-85.

(26) Camp, D.; Davis, R. A.; Evans-Illidge, E. A.; Quinn, R. J. Future Med. Chem. 2012, 4, 10671084.

(27) Koehn, F. E. Med. Chem. Commun. 2012, 3, 854-865.

(28) Cragg, G. M.; Newman, D. J.; Snader, K. M. J. Nat. Prod. 1997, 60, 52-60.

(29) Newman, D. J.; Cragg, G. M.; Snader, K. M. J. Nat. Prod. 2003, 66, 1022-1037.

(30) Newman, D. J.; Cragg, G. M. J. Nat. Prod. 2007, 70, 461-477.

(31) Newman, D. J.; Cragg, G. M. J. Nat. Prod. 2012, 75, 311-335.

(32) Cragg, G. M.; Newman, D. J. BBA-Gen. Subjects 2013, 1830, 3670-3695. 
(33) Camp, D. Drugs Fut. 2013, 38, 245-256.

(34) Butler, M. S. Nat. Prod. Rep. 2005, 22, 162-195.

(35) Butler, M. S. Nat. Prod. Rep. 2008, 25, 475-516.

(36) Butler, M. S.; Robertson, A. A. B.; Cooper, M. A. Nat. Prod. Rep. 2014, 31, 1612-1661.

(37) Vieth, M.; Siegel, M. G.; Higgs, R. E.; Watson, I. A.; Robertson, D. H.; Savin, K. A.; Durst, G. L.; Hipskind, P. A. J. Med. Chem. 2004, 47, 224-232.

(38) Hoffmann, R. W. Angew. Chem. Int. Ed. Engl. 2013, 52, 123-130.

(39) Sneader, W. Drug Discovery. A History.; John Wiley \& Sons: Chichester, West Sussex, UK, 2005.

(40) Martindale The Complete Drug Reference; 38th ed.; Brayfield, A., Ed.; Pharmaceutical Press: London, UK, 2014.

(41) Patridge, E.; Gareiss, P.; Kinch, M. S.; Hoyer, D. Drug Discov. Today 2015, http://dx.doi.org/10.1016/j.drudis.2015.01.009.

(42) Wilson, R. M.; Danishefsky, S. J. J. Org. Chem. 2006, 71, 8329-8351.

(43) Wilson, R. M.; Danishefsky, S. J. Angew. Chem. Int. Ed. 2010, 49, 6032-6056.

(44) Szychowski, J.; Truchon, J.-F.; Bennani, Y. L. J. Med. Chem. 2014, 57, 9292-9308.

(45) van de Waterbeemd, H.; Smith, D. A.; Beaumont, K.; Walker, D. K. J. Med. Chem. 2001, 44, 1313-1333.

(46) Peters, J. U.; Schnider, P.; Mattei, P.; Kansy, M. ChemMedChem 2009, 4, 680-686.

(47) Cronin, M. T. D. Curr. Comp. Aided Drug Des. 2006, 2, 405-413.

(48) Hughes, J. D.; Blagg, J.; Price, D. A.; Bailey, S.; Decrescenzo, G. A.; Devraj, R. V.; Ellsworth, E.; Fobian, Y., M.; Gibbs, M., E.; Gilles, R. W.; Greene, N.; Huang, E.; Krieger-Burke, T.; Loesel, J.; Wager, T.; Whiteley, L.; Zhang, Y. Bioorg. Med. Chem. Lett. 2008, 18, 4872-4875.

(49) Waring, M. J. Expert Opin. Drug Discov. 2010, 5, 235-248.

(50) Ritchie, T. J.; Macdonald, S. F.; Young, R. J.; Pickett, S. D. Drug Discov. Today 2011, 16, 164171.

(51) Wenlock, M. C.; Austin, R. P.; Barton, P.; Davis, A. M.; Leeson, P. D. J. Med. Chem. 2003, 46, 1250-1256.

(52) Butler, M. S.; Blaskovich, M. A.; Cooper, M. A. J. Antibiot. 2013, 66, 571-591.

(53) Walters, W. P.; Green, J.; Weiss, J. R.; Murcko, M. A. J. Med. Chem. 2011, 54, 6405-6416.

(54) Muigg, P.; Rosén, J.; Bohlin, L.; Backlund, A. Phytochem. Rev. 2013, 12, 449-457.

(55) Feher, M.; Schmidt, J. M. J. Chem. Inf. Comput. Sci. 2003, 43, 218-227. 
(56) Henkel, T.; Brunne, R. M.; Muller, H.; Reichel, F. Angew. Chem. Int. Ed. Engl. 1999, 38, 644647.

(57) Grabowski, K.; Schneider, G. Curr. Chem. Biol. 2007, 1, 115-127.

(58) Scheffé, H. The Analysis of Variance; Wiley: New York, NY, 1959 (reprinted 1999).

(59) Pardridge, W. M. Drug Discov. Today 2007, 12, 54-61.

(60) Gleeson, M. P. J. Med. Chem. 2008, 54, 817-834.

(61) Approved Drug Products with Therapeutic Equivalence Evaluations; 34th ed.; U.S. Food and Drug Administration: Washington, DC, 2014.

(62) Pryka, R. D.; Rodvold, K. A.; Rotschafer, J. C. Clin. Pharm. 1988, 7, 647-658.

(63) Ritchie, T. J.; Luscombe, C. N.; Macdonald, S. J. F. J. Chem. Inf. Model. 2009, 49, 1025-1032.

(64) Pharmaceutical Manufacturing Encyclopedia; 3rd ed.; William Andrew Publishing: Norwich, NY, 2007.

(65) Cumulative NCE Introduction Index, 1983-2013. In Annual Reports in Medicinal Chemistry; Desai, M. J., Ed.; Academic Press: San Diego, CA, 2014; Vol. 49, p 542-568.

(66) 2007 USP Dictionary of USAN and International Drug Names; 43rd ed.; U.S. Pharmacopeia: Rockville, MD, 2007.

(67) US National Library of Medicine: Bethesda, MD, 2014.

(68) SciFinder; 2014 ed.; American Chemical Society: Washington, DC, 2015.

(69) IBM SPSS Statistics for Windows, Version 22.0; IBM Corp.: Armonk, NY, 2013. 
Table of Contents graphic

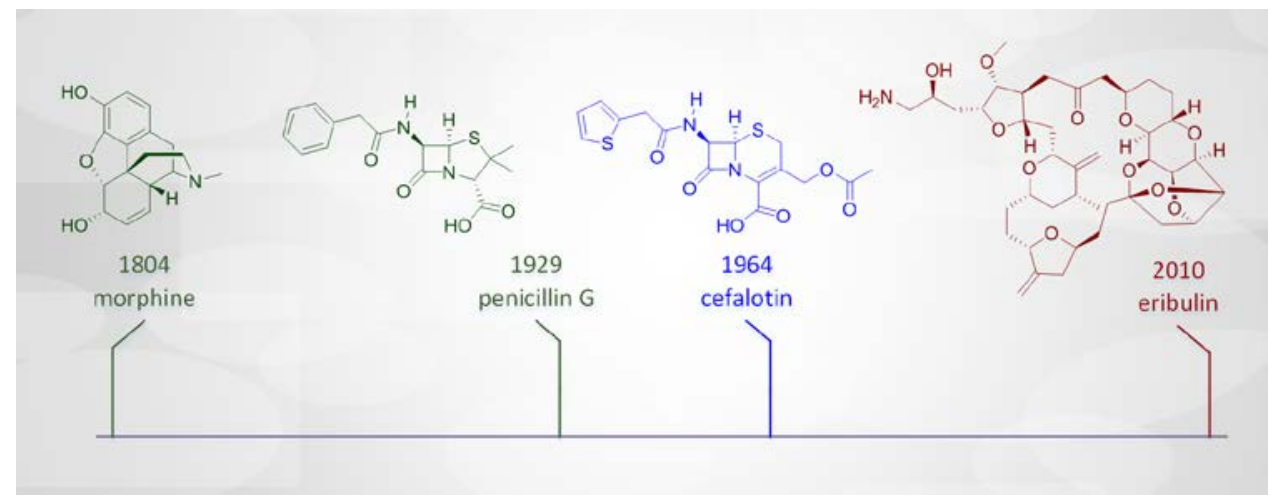

